\title{
Análise da eficiência dos sistemas estruturais para edifícios em concreto
}

\author{
An analysis of the efficiency of structural system in \\ reinforced concrete buildings
}

\begin{abstract}
Gildemir Silva
Bruno Prata

Augusto Albuquerque

Resumo

$\mathbf{N}$

os últimos anos houve uma evolução dos métodos construtivos empregados no Brasil, principalmente no que se refere às estruturas de concreto. Embora a literatura sobre eficiência na construção civil seja vasta, a proposição de métodos não paramétricos para a análise de eficiência de processos construtivos ainda é bastante limitada. Este trabalho apresenta um modelo para análise de eficiência baseado na técnica Data Envelopment Analysis (DEA) para uma análise comparada da eficiência dos sistemas estruturais de edifícios em concreto, avaliando a utilização eficiente dos insumos de produção (capital e trabalho) para a produção da estrutura. No trabalho foram comparados diversos sistemas estruturais, entre os quais pavimentos formados por lajes nervuradas apoiadas em faixas protendidas, formados por lajes apoiadas em vigas altas e formados por lajes apoiadas diretamente nos pilares, em um total de 23 obras, de 16 construtoras com reconhecida atuação no mercado imobiliário, monitoradas periodicamente, o que permite um diagnóstico do setor. O modelo proposto pode ser usado pelos planejadores com vantagens significativas. Os resultados apontam que pode haver ganhos financeiros substanciais ao melhor alocar mão de obra para o mesmo método construtivo.
\end{abstract}

Palavras-chave: Lajes. Análise envoltória de dados. Produtividade.

Gildemir Silva Universidade Federal do Ceará Fortaleza - CE - Brasil

Bruno Prata Universidade Federal do Ceará Fortaleza - CE - Brasil

Augusto Albuquerque Universidade Federal do Ceará Fortaleza - CE - Brasil

Recebido em 16/02/16 Aceito em 25/05/17

\section{Abstract}

In recent years there has been significant progress in the construction methods used in Brazil, particularly regarding concrete structures. Although the literature on efficiency in civil construction is vast, there is still very limited availability of non-parametric methods for the efficiency evaluation of constructive processes. This paper presents a mathematical model for efficiency analysis based on the Data Envelopment Analysis (DEA) technique for comparative assessment of construction methods in the production of concrete slabs. In this investigation we compared prestressed and solid slabs from a group of 16 building companies and 23 buildings produced in Fortaleza, which were periodically monitored, allowing us to perform a diagnosis of the sector. The proposed model can be used by planners, offering them significant advantages. The results indicate that there may be substantial financial gains, through better allocating labour for the same constructive method.

Keywords: Slabs. Data envelopment analysis. Productivity. 


\section{Introdução}

Nos últimos anos, a indústria da construção civil brasileira evoluiu em métodos construtivos, tendo padronizado procedimentos e alcançado uma produtividade mais elevada. Segundo a Câmara Brasileira da Construção Civil (FUNDAÇÃO..., 2012), as empresas do setor aumentaram a produtividade de 2003 a 2009 da seguinte forma: a produtividade total dos fatores (PTF), definida pela relação entre produtividade do trabalho e produtividade do capital, cresceu 3,1\%.

O relatório indica que o período foi caracterizado por uma série de aprimoramentos institucionais que fizeram a produtividade da mão de obra crescer $5,8 \%$ ao ano; já a produtividade do capital (valor adicionado/unidade de capital) foi negativa, com queda de $3,5 \%$ ao ano, indicando que o investimento realizado pelas empresas em máquinas, equipamentos e terrenos contribuiu para aumentar a produtividade do trabalho e diminuir a do capital.

Ainda segundo FGV Projetos (2012), em obras verticais o valor adicionado das empresas subgrupo edificações cresceu à notável taxa de 19,2\% ao ano, portanto superior ao crescimento do emprego $(12,8 \%$ ao ano) e ao estoque de capital por trabalhador $(7,6 \%$ ao ano). Como resultado, a produtividade total dos fatores se elevou ao ritmo de 1,9\% ao ano. Em 2006-2009, a PTF cresceu à taxa de $5,0 \%$ ao ano, com o produto crescendo a uma taxa maior que a do emprego e com o estoque de capital físico acompanhando o crescimento do emprego.

O relatório, de uma forma geral, aponta para um ganho de produtividade decorrente da capacitação dos trabalhadores e pouco em investimento em máquinas e equipamentos.

Diversos trabalhos têm apresentado análises de eficiência na construção civil, alguns centrados no desempenho das empresas, como Araújo Júnior, Nogueira e Shikida (2012), ou pré-qualificação de construtores, como McCabe, Tran e Ramani (2005), enquanto outros avaliam materiais empregados e a ecoeficiência das edificações, como Tatari e Kucukvar (2012) e Brondani, Mohamad e Isaia (2015).

Dantas (2011) e Calçada (2014) analisam processos construtivos com vistas a estabelecer um benchmark no setor da construção civil. Além desses autores, Berr e Formoso (2012) apresentam um comparativo entre processos construtivos para habitações sociais e analisam a construção em sua totalidade, não um comparativo de um processo em relação a outro para uma fase da construção.
Nota-se nos textos estudados uma preocupação profissional e acadêmica em avaliar a construção e as empresas de forma agregada, mas a avaliação de processos produtivos na construção civil é incipiente. Portanto, não se observou preocupação em responder ao seguinte questionamento: o aumento da produtividade ou diferencial das empresas não teve ganhos decorrentes dos novos processos produtivos, das padronizações nos canteiros e da melhor uniformização na indústria?

Assim, este trabalho se propõe a responder ao questionamento acima mediante a análise do processo de execução das estruturas de concreto de edificações verticais, comparando o ganho na adoção de um sistema estrutural ante outro. Para tanto, é descrita a relação insumo-produto para os diferentes métodos e modelada a produção, de forma não paramétrica, utilizando a técnica Análise Envoltória de Dados (Data Envelopment Analysis DEA).

Toda análise foi efetuada a partir de visitas periódicas e contato com os engenheiros residentes e projetistas, para levantamento de dados (funcionários, tempo e insumos), de 23 obras, de edifícios residenciais na cidade de Fortaleza, os quais estão sendo executados por 16 construtoras.

Trata-se de projetos com vãos livres entre $6,0 \mathrm{~m} \mathrm{e}$ $8,5 \mathrm{~m}$, a maioria com em torno de 23 pavimentos, que são valores muito representativos do mercado imobiliário para esse setor.

\section{Teoria da produção e mensuração de eficiência}

Analisar a produtividade de uma unidade de uma planta de produção em que não haja grandes diferenças de comportamento quanto ao objetivo, principalmente em aspectos de custo e método para alcançar o produto, dita homogênea (Decision Making Unit-DMU), exige que três decisões sejam corretamente tomadas:

(a) dentro de todos os níveis de produção possíveis, deve-se escolher o que maximize os benefícios;

(b) de todos os insumos para o nível escolhido, deve-se escolher o conjunto de insumos que minimize os custos; e

(c) a empresa deve produzir com o mínimo de insumos possível.

Desses três pressupostos decorrem três possibilidades de eficiência: eficiência de escala (produção em uma escala de tamanho ótimo); eficiência alocativa (conjunto de insumos que 
minimiza custos); e eficiência técnica (máxima produção possível com o conjunto de insumos empregado).

Coelli et al. (1998) reduzem essa abordagem em eficiência técnica com duas componentes: a componente puramente técnica, que se refere à habilidade de evitar desperdícios na produção, e a componente alocativa ou preço, que se refere à habilidade de combinar os insumos e produtos em proporções ótimas, dados os preços deles. A curva de possibilidade de produção representa o máximo que pode ser produzido com base nos insumos disponíveis. Portanto, no sentido pareteano, implica maior produção de um bem sem reduzir a produção de outros, isto é, quando a economia se encontra sobre sua curva de possibilidade de produção.

Para unidades homogêneas essa é uma das chaves para verificar possibilidade de ganhos de produção. Na Figura 1 são ilustradas as definições de produtividade, eficiência técnica, fronteira de produção e economias de escala.

A fronteira de produção é representada na Figura 1 pela linha vermelha “0D” e representa a produção máxima para cada nível de insumo, o que reflete o estado da tecnologia na indústria. A empresa é tecnicamente eficiente se está na fronteira de produção, sendo ineficiente se estiver abaixo da curva. Por exemplo, o ponto "A” é tecnicamente ineficiente, e os pontos " $B$ " e "C" são eficientes.

Pode-se observar que " $A$ " pode alcançar " $B$ " sem aumentar a quantidade de insumos, pois em " $\mathrm{A}$ " está se produzindo ineficientemente. Admitindo que não existem valores de produtos e insumos negativos, todos os pontos abaixo da curva são factíveis, porque, dada uma combinação de insumos, pode-se produzir algo. Contudo, acima da curva não se pode produzir com o insumo relativo, pois ultrapassaria a fronteira de produção da indústria.

Na Figura 2 é possível verificar a diferença entre produtividade e eficiência técnica, pois a inclinação relativa da produção nos pontos “ $A$ ” e "B" são diferentes, embora com mesmos insumos. No ponto "C" a reta é tangente à fronteira de produção. Assim, tecnicamente "C" é um ótimo, pois é a tangente à curva “ $0 \mathrm{D}$ ” com declividade máxima, "B" está na fronteira, não é um ótimo, mas é mais produtivo que “A”. O ponto “ $C$ ” é um ótimo porque tem melhor escala de produção (combinação insumo/produto).

Temporalmente esse conceito é diferente, pois, ao se explorar a temporalidade, tem-se o conceito de mudanças técnicas que são possíveis de um período para outro, implicando a mudança ou a forma da fronteira de produção com ampliação ou redução do conjunto de produção conforme ilustrado pela linha azul " $0 \mathrm{E}$ ".

O crescimento em produtividade é consequência, conforme Coelli, Rao e Battese (1998), das melhoras em eficiência, mudanças técnicas ou economias de escala, podendo ainda ser resultado de uma combinação desses fatores.

Segundo Silva et al. (2011) existem vários métodos computacionais para medir produção e eficiência, podendo ser destacados modelos econométricos de funções de produção, índices de PTF, DEA e fronteira estocástica. Para um aprofundamento dessas técnicas, recomenda-se consulta a Coelli et al. (2003).

\section{Figura 1 - Fronteira de produção, eficiência técnica, economia de escala e produtividade}

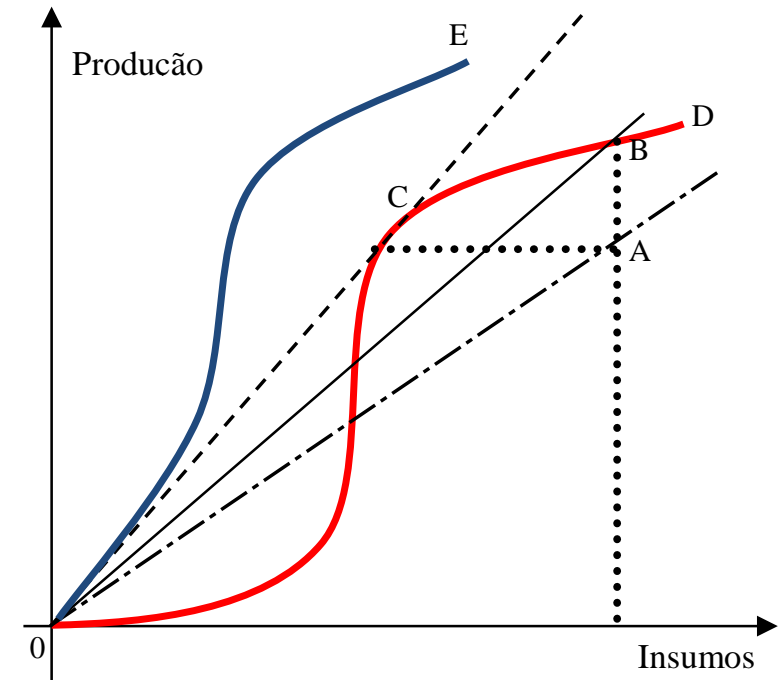


Conforme Charnes et al. (1994), a Análise Envoltória de Dados é uma técnica de avaliação da eficiência relativa entre unidades produtivas que executam as mesmas tarefas e possuem objetivos semelhantes, utilizando múltiplos produtos e múltiplos insumos. Esse método trabalha com a otimização individual de cada observação, tendo como objetivo calcular uma fronteira eficiente determinada pelas unidades que são Paretoeficientes.

A técnica DEA fornece três resultados:

(a) a identificação de um conjunto de unidades eficientes (que determinam a fronteira de eficiência);

(b) a medida de ineficiência para cada unidade que não está sobre a fronteira (a distância da fronteira representa a potencialidade de crescimento da produtividade); e

(c) as taxas de substituição (pesos) que determinam cada região da fronteira de eficiência e caracterizam as relações de valor que permitem a classificação dessa região como eficiente.

Para a aplicação da técnica DEA, conforme Silva et al. (2011), seguem-se os seguintes passos:

(a) verificação da coerência das variáveis para input e output, considerando as hipóteses básicas e realizando testes estatísticos;

(b) determinação da fronteira de eficiência a partir do cálculo da eficiência; e

(c) criação de um valor virtual de input e output de cada observação para se medir o quão distante esse ponto se encontra da fronteira de eficiência.

Para aferição significativa da eficiência, é necessário garantir que:

(a) as unidades sob análise sejam comparáveis, ou seja, realizem as mesmas tarefas e possuam objetivos semelhantes;

(b) as unidades atuem sob as mesmas condições de mercado; $\mathrm{e}$

(c) as variáveis, isto é, os inputs e os outputs das unidades, sejam as mesmas, exceto em intensidade ou magnitude.

A eficiência é calculada obtendo-se uma relação entre o vetor de outputs e de inputs, estando situada entre zero e um, representada pela Equação 1:

$\theta=\frac{u_{0}+u_{1} y_{1}+\ldots+u_{n} y_{n}}{v_{0}+v_{1} x_{1}+\ldots+v_{n} x_{n}} \leq 1,0$

Em que:

$\vec{y}=\left(y_{1}, y_{2}, \ldots, y_{n}\right)$ é o vetor de inputs; $\vec{x}=\left(x_{1}, x_{2}, \ldots, x_{n}\right)$ é o vetor de outputs;

$u_{i}$ são os pesos dos inputs; e

$v_{i}$ os pesos dos outputs.

Os primeiros modelos DEA desenvolvidos foram o CRS (Constant Return to Scale - Retornos Constantes de Escala) ou CCR (abreviatura dos nomes de seus criadores Charnes, Cooper e Rhodes (1978)) e VRS (Variable Return to Scale - Retornos Variáveis de Escala) ou BCC (abreviatura de Banker, Charnes e Cooper (1984)).

\section{Sistemas estruturais para edifícios em concreto e aspectos de produtividade}

Várias são as alternativas estruturais em concreto, moldado no local, para edifícios de múltiplos pavimentos devido a variações tais como tipos de lajes, existência de vigas e utilização da protensão.

As lajes mais utilizadas para essas aplicações são as lajes maciças e nervuradas, que utilizam cubetas plásticas removíveis. A possibilidade de as lajes se apoiarem diretamente sobre os pilares, sem a presença de vigas, possibilita o surgimento das chamadas lajes lisas (sem capiteis junto aos pilares) e das lajes cogumelo (com capiteis), nomenclatura de acordo com a NBR 6118 (ABNT, 2014).

A protensão é um recurso bastante utilizado, em vigas ou lajes, maciças ou nervuradas, que facilita o controle das deformações dos pavimentos. As variantes provocadas por essas combinações entre tipos de elementos estruturais, utilização de vigas ou não para apoio de lajes, e utilização da protensão, caracterizam os sistemas estruturais. Cabe ao projetista de estruturas conceber um sistema estrutural que melhor atenda a cada empreendimento, obedecendo aos critérios de segurança (ELU) e de serviço (ELS), observando os requisitos prescritos na NBR 6118 (ABNT, 2014) e NBR 15575 (ABNT, 2013), além de buscar o melhor resultado financeiro.

No início do século XX a maioria dos prédios em concreto no Brasil eram compostos de pavimentos formados por lajes maciças apoiadas em vigas. Esse panorama começou a se modificar nos anos 80, com maior utilização de lajes nervuradas, que apresentam maior autonomia de vãos devido a suas maiores inércias com menor consumo. Os anos 90 são marcados pela popularização das lajes nervuradas e pela maior utilização dos sistemas sem vigas (lajes lisas), e, no final da década, pelo início da utilização da protensão não aderente. A protensão possibilitou a utilização de vãos ainda maiores e impulsionou os sistemas sem viga, além 
de possibilitar o desenvolvimento de um novo sistema estrutural, que é o pavimento formado por lajes nervuradas apoiadas em vigas faixa protendidas (vigas com a mesma altura das lajes).

Toda essa evolução sempre buscou o aumento dos vãos, priorizando formas com poucos recortes, ou seja, com poucas vigas internas ao pavimento. Isso garante maior facilidade de execução e maior flexibilidade para o leiaute dos apartamentos do ponto de vista arquitetônico. Além disso, vãos maiores aumentam a produtividade de execução dos pavimentos, diminuindo o ciclo de forma, montagem, concretagem e desforma.

É forte a tendência de sistemas estruturais com ausência de recortes internos (vigas) nas formas, tais como sistemas com laje lisa ou com lajes nervuradas apoiadas em faixas, devido à facilidade de execução, pois são mais fáceis de confeccionar e de se posicionar a armadura, além da facilidade de montagem das formas.

O processo produtivo de fabricação de pavimentos em concreto, moldado in loco, é bem definido em seus insumos produtivos, sendo essencialmente composto de mão de obra e capital, mudando apenas o processo construtivo no que tange à tecnologia empregada, o que implicará o uso de formas de madeira ou de plástico, escoras de madeira ou de metal e maior precisão no nivelamento. Esses processos que se diferenciam de um sistema estrutural para outro podem resultar em perdas ou ganhos de eficiência, os quais apenas podem ser avaliados de forma comparada e isenta de viés.

\section{Comparação da eficiência produtiva de diferentes sistemas estruturais em concreto empregados em edificações verticais}

Analisar uma unidade dentro de outra maior (processo de produção da estrutura de concreto dentro da construção de edificações verticais), sendo o processo de produção mais homogêneo nos insumos, acarreta que as estimativas, paramétricas ou não paramétricas, serão mais robustas, mesmo em pequenas amostras (WOOLDRIDGE, 2011; GUJARATI; PORTER, 2011).

O processo de execução da estrutura pode ser considerado homogêneo, pois faz parte de um mesmo centro de custo, o que exige materiais e mão de obra uniformemente distribuídos para períodos múltiplos do ciclo da laje, implicando poucos custos indiretos que necessitem de rateio. Portanto, análises paramétricas ou não paramétricas, mesmo empregando pequenas amostras, terão boa robustez nos resultados. Entende-se por ciclo da laje como o período entre o término da concretagem de um pavimento e a conclusão da concretagem do pavimento imediatamente acima.

Na análise apresentada neste trabalho são empregadas estimativas paramétricas para avaliar retornos de escala, seguindo com estimativas não paramétricas para mensurar a distância das unidades analisadas da eficiência máxima possível para o processo, bem como indicar os ajustes nos insumos que levariam as unidades tomadoras de decisão para a fronteira de eficiência.

\section{Modelo e variáveis utilizadas}

Os dados utilizados neste estudo advêm de um levantamento realizado em 23 obras que utilizam quatro diferentes sistemas estruturais: pavimento formado prioritariamente por lajes nervuradas apoiadas em vigas faixas protendidas, pavimento em laje maciça sem vigas internas (exceto região de escada e elevadores), pavimento em laje maciça sem vigas internas (exceto região de escada e elevadores) e de borda, e pavimento formado prioritariamente por lajes nervuradas apoiadas em vigas altas protendidas de borda.

Esses edifícios se encontravam em execução no ano de 2016, de forma que já foram projetados atendendo à norma de desempenho NBR 15575 (ABNT, 2013). Ressalta-se também que foram projetados por escritórios com atuação nacional, bem como foram construídos por construtoras de elevado conceito no mercado imobiliário, tendo inclusive algumas delas empreendimentos em várias cidades do Norte e do Nordeste.

Neste trabalho é analisada a produtividade proporcionada por esses quatro sistemas estruturais identificados, que foram utilizados no conjunto de obras aqui reunido. O diferencial entre os sistemas estruturais do ponto de vista da produção se dá principalmente na facilidade de execução da forma de madeira e da confecção e posicionamento da armadura, além da concretagem que alguns têm em relação a outros em função de uma geometria mais simples, ou seja, com menos reentrâncias (recortes de forma). Essa facilidade implica um uso menos intensivo de mão de obra, impactando na produtividade e na eficiência do processo construtivo.

As variáveis envolvem quantidades e salários dos vários profissionais participantes no processo produtivo, tipo de forma, área produzida, ciclo da laje, quantidade de pavimentos produzidos por mês e custo de capital empregado em cada pavimento 
por mês, resumidos nas siglas das colunas na Tabela 1 como:
(a) número de serventes (X.SERV);
(b) número de auxiliares (X.AUX);
(c) número de profissionais (X.PROF);
(d) número de encarregados (X.ENCAR);
(e) número de mestres (X.MESTRE);
(f) área do pavimento em metro quadrado (AREA_M2);

(g) ciclo da laje em dias (CICLO_LJ_DIAS);

(h) número de lajes por mês (N_LJ_MES);

(i) custo por dia (CUSTO_DIA);

(j) homem por dia (HOM_DIA);

(k) área por dia (M2_DIA);

(l) custo unitário (CUSTO_UN);

(m) área de forma viga e pilar (FORM_VIGA_PILAR);

(n) área de forma de viga (FORM_VIGA); e

(o) custo por metro quadrado (CUSTO_R_M2).

O resumo estatístico das variáveis é exposto na Tabela 1, indicando média, desvio padrão, moda, máximo e mínimo de cada variável. Os salários praticados não foram indicados, pois não existia variação entre empresas, sendo $\mathrm{R} \$ 1.366,54, \mathrm{R} \$$ 1.544,09, R\$ 2.075,80, R\$2.446,61 e R\$ 3.609,00 para serventes, auxiliar, profissional (carpinteiro, pedreiro e ferreiro), encarregado e mestre de obras respectivamente.

Com base na amostra analisada, foi possível observar que os pavimentos produzidos tinham áreas entre 272 e 1.392 metros quadrados, havendo grande variação da quantidade de produto a ser executado. No que concerne à mão de obra, esta apresenta variações entre auxiliares e serventes de 0 a 17 funcionários, para cargos intermediários entre serventes e encarregados, daqui em diante chamados de profissionais, em um intervalo de 8 a 29 funcionários, e entre encarregados e mestres de obra, uma variação entre 0 ao máximo de 2 por obra.

O custo unitário dos materiais e equipamentos utilizados para a construção oscila entre R\$ 15,75 e $\mathrm{R} \$ 85,25$, o que reflete um emprego diferente do capital na construção. Avaliando as medidas de produção pela média e o desvio padrão amostral, os resultados refletem oscilação na produção e produtividade, seja em termos do custo por dia, número de funcionários por dia, número de pavimentos por mês e ciclo de lajes em dias, o qual contempla a montagem das formas, o escoramento e a concretagem total do pavimento.

Tabela 1 - Estatística descritiva das variáveis

\begin{tabular}{l|c|c|c|c|c}
\hline & X.SERV & X.AUX & X.PROF & X.ENCAR & X.MESTRE \\
\hline Média & 5,62 & 8,85 & 15,81 & 1,46 & 0,90 \\
Desvio padrão & 4,70 & 4,85 & 6,63 & 0,63 & 0,37 \\
Moda & 3,00 & 7,00 & 8,00 & 2,00 & 1,00 \\
Máximo & 17,00 & 17,00 & 29,00 & 2,00 & 2,00 \\
Mínimo & 0,00 & 0,00 & 8,00 & 0,00 & 0,00 \\
\hline & AREA_M2 & CICLO_LJ_DIA & N_LJ_MES & CUSTO_DIA & HOM_DIA \\
\hline Média & 515,49 & 7,48 & 2,96 & 2904,84 & 3,25 \\
Desvio padrão & 227,08 & 1,97 & 0,62 & 839,74 & 0,90 \\
Moda & 550,00 & 7,00 & 3,00 & \#N/DISP & \#N/DISP \\
Máximo & 1392,00 & 14,00 & 4,20 & 4517,46 & 4,68 \\
Mínimo & 272,28 & 5,00 & 1,50 & 1756,02 & 1,41 \\
\hline & M2_DIA & CUSTO_UN & FORM_VIGA_PILAR & FORM_VIGA & CUSTO_R_M2 \\
\hline Média & 74,68 & 44,87 & 0,88 & 0,40 & 233,06 \\
Desvio padrão & 48,01 & 15,69 & 0,26 & 0,20 & 20,61 \\
Moda & 78,57 & \#N/DISP & 0,78 & \#N/DISP & 230,00 \\
Máximo & 278,40 & 85,26 & 1,37 & 0,86 & 280,00 \\
Mínimo & 21,14 & 13,76 & 0,43 & 0,03 & 200,00 \\
\hline
\end{tabular}




\section{Modelando o processo produtivo}

A forma funcional para a estimativa paramétrica da função de produção foi a função clássica CobbDouglas, pois ela tem características largamente estudadas na literatura, entre as quais a parcimônia e a precisão em descrever a função de produção de processos produtivos. A estimativa foi por Mínimos Quadrados Ordinários, uma vez que a função de produção pode ser linearizada e não apresenta nenhuma anomalia econométrica na estimativa. A função foi linearizada pela transformação das variáveis em logaritmos. Assumiu-se $\beta_{0}=0$, pois não existe produção da estrutura no caso de não se empregar capital nem trabalho.

O modelo foi rodado no software (R), apresentando boa aderência, com coeficiente $R^{2}$ ajustado de 0,9966 e estatísticas $F$ e ts significantes a 5\%. A taxa marginal de substituição entre capital e trabalho indica que se pode reduzir uma unidade de capital toda a vez que se reduz 0,025 de trabalhadores, valendo o inverso para a taxa marginal de substituição entre trabalho e capital (aumentar uma unidade de pessoas implica o aumento de 1,02 de capital empregado). Nota-se que o fator trabalho aumenta a ineficiência da produção.

O resultado do modelo indica possíveis retornos crescentes de escala, uma vez que o somatório dos parâmetros é maior que 1 e ambos são significantes a 5\%. Assumem-se retornos crescentes para a modelagem não paramétrica da produção utilizando o modelo BCC-DEA, sem orientação, conforme equações descritas a seguir (Equação 2).

$\min \theta-\varepsilon\left(\sum_{i=1}^{m} s_{i}^{-}+\sum_{r=1}^{s} s_{r}^{+}\right)$

Eq. 2

Sujeito a:

$\sum_{j=1}^{n} x_{i j} \lambda_{j}+s_{i}^{-}=\theta_{o} x_{i o} \quad i=1,2, \ldots, m$;

$\sum_{j=1}^{n} y_{r j} \lambda_{j}-s_{r}^{+}=y_{r o} \quad r=1,2, \ldots, s_{;}$

$\sum_{j=1}^{n} \lambda_{j}=1$

$\lambda_{j}, s_{i}^{-}, s_{r}^{+}$

Cuja forma dual é expressa na Equação 3:

$\max z=\sum_{r=1}^{s} u_{r} y_{r o}-u_{o}$

Sujeito a:

$\sum_{r=1}^{s} u_{r} y_{r j}-\sum_{i=1}^{m} v_{i} x_{i j}-u_{o} \leq 0 \quad j=$

$1, \ldots, n$,

$\sum_{i=1}^{m} v_{i} x_{i o}=1$

$v_{i} \geq \varepsilon, u_{r} \geq \varepsilon, u_{0}$ livre em sinal

E a projeção na fronteira de eficiência se dá pelas Equação 4: $\left\{\begin{array}{c}\hat{x}_{i o}=\theta_{o}^{*} x_{i o}-s_{i}^{-*}=\sum_{j=1}^{n} x_{i j} \lambda_{j}^{*}, i=1, \ldots, m, \\ \hat{y}_{r o}=y_{r o}+s_{r}^{+*}=\sum_{j=1}^{n} y_{r j} \lambda_{j}^{*}, r=1, \ldots, s,\end{array} \quad\right.$ Eq. 4

Para retornos crescentes, decrescentes ou constantes de escala, implementam-se os seguintes critérios:

(a) retornos crescentes em $\left(\hat{x}_{o}, \hat{y}_{o}\right)$ - se e somente se $u_{0} *<0$ para todas as soluções ótimas;

(b) retornos decrescentes - prevalece em $\left(\hat{x}_{o}, \hat{y}_{o}\right)$ se e somente se $u_{o}^{*}>0$ para todas as soluções ótimas; e

(c) retornos constantes - prevalece em $\left(\hat{x}_{o}, \hat{y}_{o}\right)$ se e somente se $u_{o} *=0$ para pelo menos uma solução ótima.

A estimativa não paramétrica não possui restrições quanto ao número de variáveis a serem utilizadas e quanto ao tamanho da amostra, pois não se perdem graus de liberdade, nem se avaliam parâmetros estatísticos do resultado do modelo.

O modelo DEA tem a finalidade de medir quão distante as amostras estão da fronteira e quão seria a expansão ou contração dos insumos para chegar à fronteira. Assim, utilizou-se no modelo DEA a divisão da mão de obra pelo número de operários por suas especialidades em cada obra: serventes, auxiliar, profissional, encarregado e mestre, e o capital foi o mesmo utilizado na estimativa paramétrica como outro insumo. A produção é a quantidade de metros quadrados de pavimentos produzidos por mês.

A relação trabalho-mão de obra versus quantidade de metros quadrados de pavimentos é apresentada na Figura 2. Observam-se empresas no limite da eficiência e um grande grupo de DMUs aquém da fronteira das possibilidades de produção, portanto possuindo margem para melhor alocação de sua mão de obra, o que, com as análises que seguem, será quantificado. As outras DMUs apresentam-se bem abaixo da curva. Ao modelar o processo como retornos variáveis, existe a possibilidade de que a DMU destacada seja um outlier, mas esta não será tratada como outlier na presente modelagem.

Os resultados do Modelo BCC-DEA sem orientação levaram aos índices de eficiência apresentados na Tabela 2. A média de eficiência das amostras indicou valores de eficiência entre 63\% e 100\%. Das 23 DMUs analisadas, 14 apresentaram eficiência $100 \%$, e 5 com eficiência menor do que $90 \%$. Analisando as obras em função de seus sistemas estruturais, tem-se que as obras com vigas faixas protendidas e lajes nervuradas apresentaram em média 89\% de eficiência; já as obras em laje lisa com viga de borda apresentaram eficiência maior, em torno de $30 \%$ se comparadas com o primeiro grupo; as em laje lisa sem viga de borda tiveram 
média de 28\%, e as estruturas com viga alta protendida de borda e lajes nervuradas foram em média 30\% mais eficientes que o grupo 1 .

Analisando os insumos empregados no processo construtivo quanto aos valores médios passíveis de redução no conjunto de obras analisadas, tem-se o resumo estatístico apresentado na Tabela 3 e as Figuras 3 a 6 para serventes, auxiliares, encarregados e mestres que apresentam a folga nas DMUs. Nota-se que a redução para alcance de eficiências melhores seria na redução de operários na seguinte ordem: profissionais, auxiliares, serventes, encarregados e mestres.

Figura 2 - Fronteira de produção da amostra de 23 obras de Fortaleza, onde $Y$ representa metros quadrados das lajes e $X$ a relação entre trabalho e mão de obra

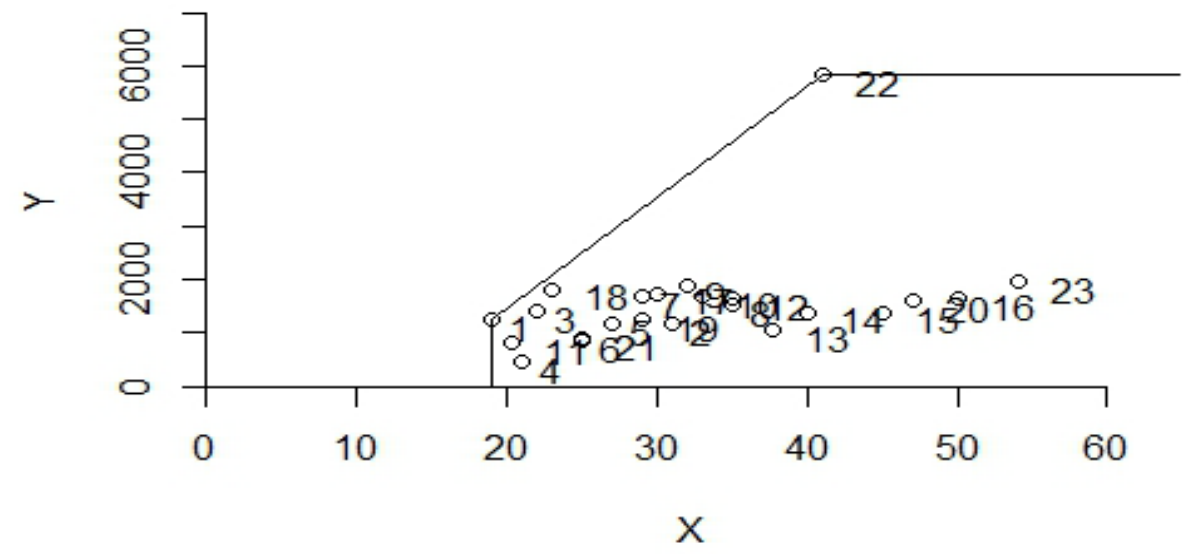

Tabela 2 - Eficiência das DMUs analisadas (empresa-obra)

\begin{tabular}{c|c|c}
\hline Obra & Construtora & Sistema estrutural \\
\hline 1 & A & 1 \\
2 & B & 1 \\
3 & C & 3 \\
4 & D & 1 \\
5 & E & 4 \\
6 & F & 4 \\
7 & G & 3 \\
8 & H & 2 \\
9 & I & 1 \\
10 & I & 1 \\
11 & I & 1 \\
12 & I & 1 \\
13 & I & 1 \\
14 & J & 3 \\
15 & J & 3 \\
16 & J & 3 \\
17 & K & 1 \\
18 & L & 2 \\
19 & L & 3 \\
20 & M & 1 \\
21 & N & 1 \\
22 & O & 1 \\
23 & P & 1 \\
\hline
\end{tabular}

Nota: Legenda:

Sistema estrutural:

1 - Viga faixa protendida com lajes nervuradas;

2 - Laje lisa com viga de borda;

3 - Laje lisa sem viga de borda; e

4 - Viga alta protendida de borda e lajes nervuradas. 
Tabela 3 - Resumo estatístico dos possíveis aj ustes para melhorar a eficiência do conjunto de obras analisadas

\begin{tabular}{l|c|c|c|c|c|c}
\hline & X.SERV & X.AUX & X.PROF & X.ENCAR & X.MESTRE & POTSALV_K \\
\hline Média & 1,75 & 2,46 & 4,52 & 0,36 & 0,19 & 55,53 \\
Desvio Padrão & 2,01 & 2,69 & 3,97 & 3,98 & 0,29 & 43,88 \\
Moda & 0,00 & 0,00 & 0,00 & 0,00 & 0,00 & 0,00 \\
Máximo & 9,54 & 8,00 & 16,27 & 1,12 & 0,56 & 140,24 \\
Minimo & 0,00 & 0,00 & 0,00 & 0,00 & 0,00 & 0,00 \\
\hline
\end{tabular}

Figura 3 - Utilização de serventes e folga de necessidade

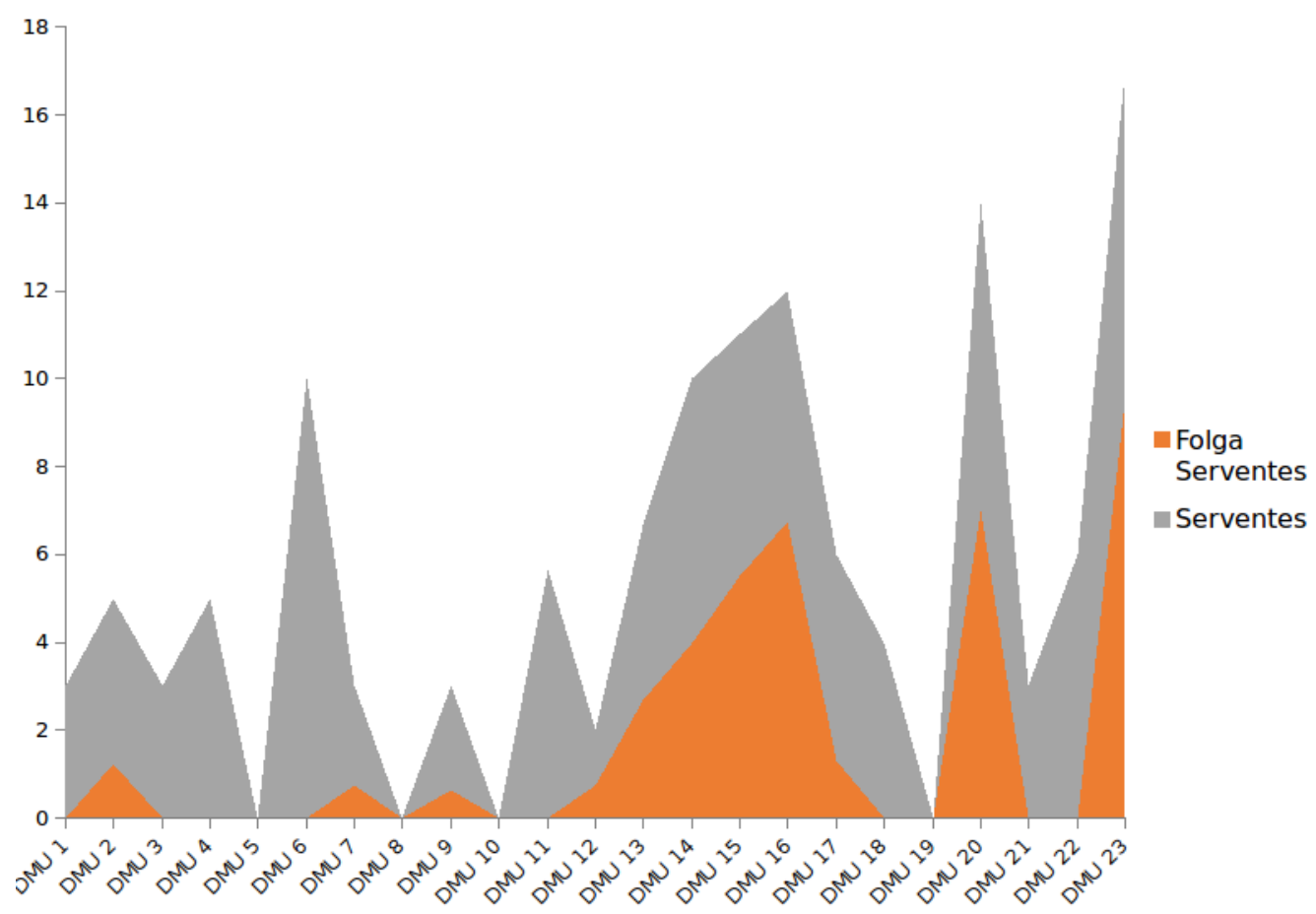

Figura 4 - Utilização de auxiliares e folga de necessidade

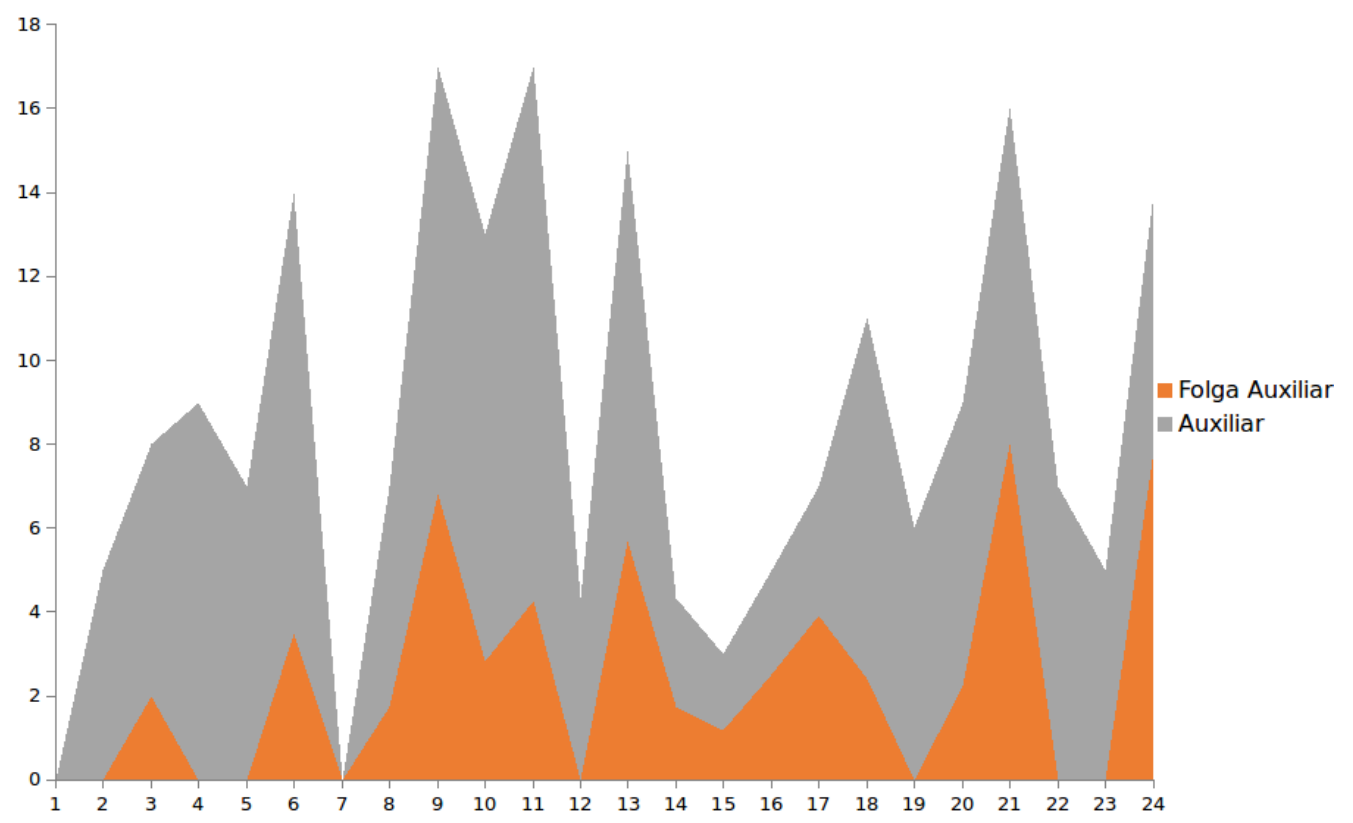


Figura 5 - Utilização de encarregados e folga de necessidade

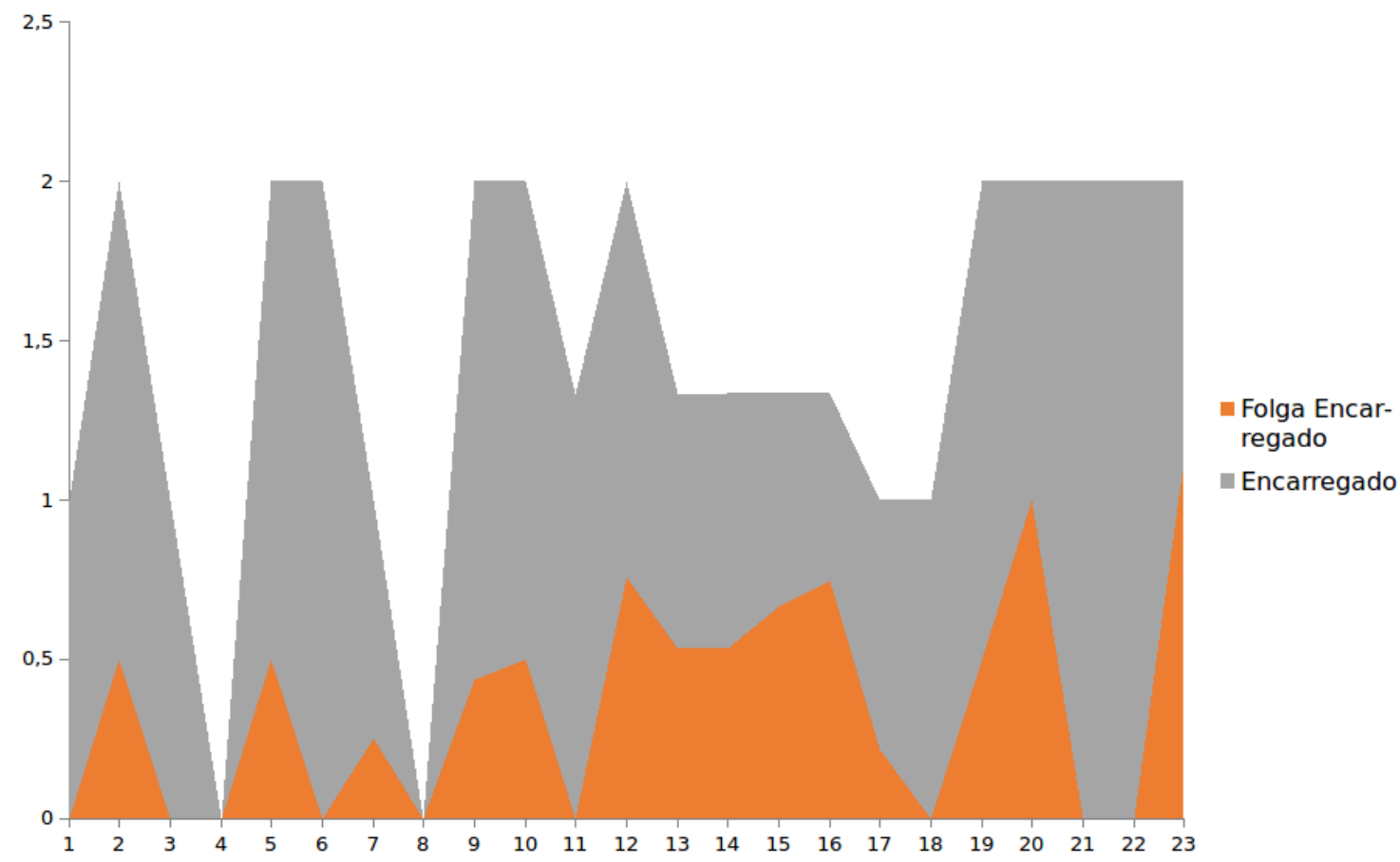

Figura 6 - Utilização de mestres e folga de necessidade

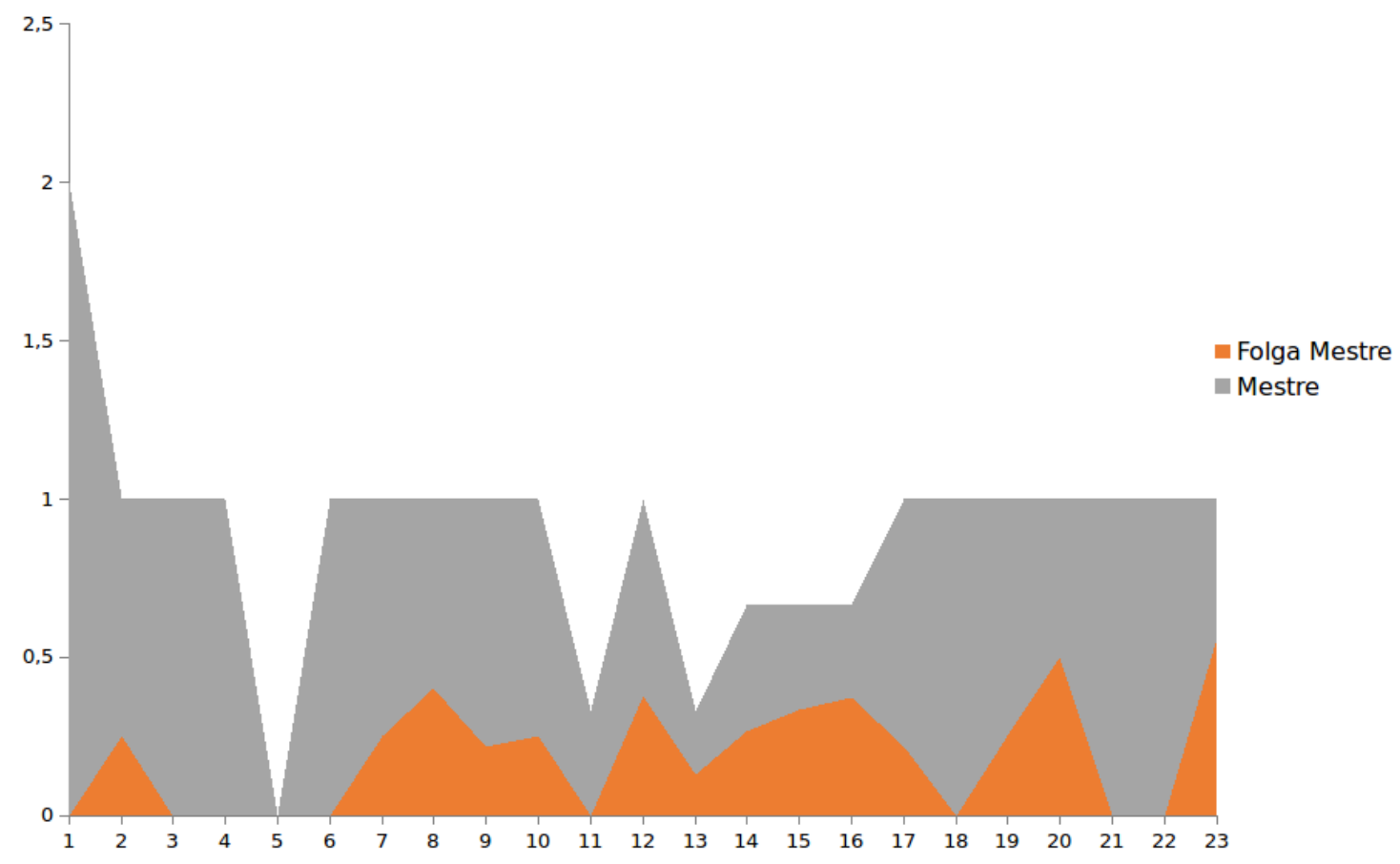

Deve-se observar que essa análise é simplificada, sendo necessário um acompanhamento contínuo das obras para compreender melhor a causa de uma ociosidade média de mão de obra nos processos produtivos analisados.

Nas Tabelas 4 e 5 são apresentadas análises individuais de cada obra para apontar melhorias nos processos avaliados. Na Tabela 4 o degradê está disposto da seguinte forma: vermelho (não há ou há pouca necessidade de ajustes), verde (há muita necessidade de ajustes) e amarelo ou laranja (há necessidade de ajustes em nível intermediário). Pode-se constatar que a construtora "I" possui poucos ajustes no que se refere ao insumo trabalho, mas ajustes em alguns pontos no emprego do 
capital. Por sua vez, a construtora “J” necessita de grandes ajustes em capital e trabalho. Já a construtora "P", para sua obra na amostra, precisaria de um ajuste em todos os insumos para a produção da estrutura.

Na Tabela 5 adotou-se o mesmo processo de ajuste por cores da Tabela 4. Verifica-se que o sistema estrutural 1 precisa ajustes tanto no capital como no trabalho, e o sistema estrutural 4 precisa de ajustes em quase todos os insumos produtivos de trabalho e menor intensidade no capital. Os sistemas estruturais 2 e 3 , embora com necessidade de pouco ajuste no insumo trabalho, são intensivos em capital. Entretanto, como foi visto na estimativa paramétrica, é melhor substituir trabalhador por capital do que o inverso, logo a estratégia de quem utiliza os sistemas estruturais 2 e 3 precisa de ajustes, porém menores do que nos outros dois processos. O sistema estrutural 2 é o mais eficiente e o com melhor emprego da mão de obra, portanto pode ser utilizado como benchmark para a indústria da construção civil no Estado do Ceará.
Uma ordenação dos sistemas estruturais analisados com base no critério de eficiência adotado indica que o sistema estrutural que melhor utiliza seus insumos seria o sistema estrutural 2; o segundo seria o sistema estrutural 4; o terceiro seria o sistema estrutural 1; e, por fim, o sistema estrutural 3, embora eficiente, seria o que pior utiliza seus insumos em média.

Com uma análise financeira do processo adotado, optar pelo sistema 2 ante o sistema 1 gera um retorno financeiro mensal de aproximadamente $\mathrm{R} \$$ 2.435,00 em serventes, de R\$ 202,52 em profissionais e de $\mathrm{R} \$ 532,40$ em encarregados, um gasto extra em auxiliar de R\$ 994,14, e para mestre de R \$ 9,77, portanto utilizar o sistema 2 comparado com o sistema 1 gera uma renda líquida adicional aproximada de R\$ 2.166,00 mensais. Fazendo o mesmo exercício comparando o sistema 3 com o 1 e o 4 com o 1 tem-se $\mathrm{R} \$ 6.727,00$ (perda) e $\mathrm{R} \$$ 7.120,00 (ganho) respectivamente.

Tabela 4 - Potenciais de redução de insumos por obra

\begin{tabular}{c|c|c|c|c|c|c|c|c|c}
\hline Obra & Construtora & Eficiência & $\begin{array}{c}\text { Sistema } \\
\text { estrutural }\end{array}$ & X.SERV & X.AUX & X.PROF & X.ENCAR & X.MESTRE & POTSALV_K \\
\hline 1 & A & 1,00 & 1 & 0,00 & 0,00 & 0,00 & 0,00 & 0,00 & 0,00 \\
\hline 2 & B & 0,72 & 1 & 1,25 & 2,00 & 3,75 & 0,50 & 0,25 & 65,00 \\
\hline 3 & C & 1,00 & 3 & 0,00 & 0,00 & 0,00 & 0,00 & 0,00 & 0,00 \\
\hline 4 & D & 1,00 & 1 & 0,00 & 0,00 & 0,00 & 0,00 & 0,00 & 0,00 \\
\hline 5 & E & 1,00 & 4 & 0,00 & 3,50 & 2,75 & 0,50 & 0,00 & 65,00 \\
\hline 6 & F & 1,00 & 4 & 0,00 & 0,00 & 0,00 & 0,00 & 0,00 & 0,00 \\
\hline 7 & G & 1,00 & 3 & 0,75 & 1,75 & 4,25 & 0,25 & 0,25 & 60,00 \\
\hline 8 & H & 1,00 & 2 & 0,00 & 6,80 & 6,80 & 0,00 & 0,40 & 92,00 \\
\hline 9 & I & 0,85 & 1 & 0,65 & 2,83 & 2,83 & 0,43 & 0,22 & 49,57 \\
\hline 10 & I & 1,00 & 1 & 0,00 & 4,25 & 3,25 & 0,50 & 0,25 & NA \\
\hline 11 & I & 1,00 & 1 & 0,00 & 0,00 & 0,00 & 0,00 & 0,00 & 0,00 \\
\hline 12 & I & 0,75 & 1 & 0,76 & 5,69 & 5,69 & 0,76 & 0,38 & 87,24 \\
\hline 13 & I & 1,00 & 1 & 2,67 & 1,73 & 10,00 & 0,53 & 0,13 & 94,40 \\
\hline 14 & J & 1,00 & 3 & 4,00 & 1,20 & 10,00 & 0,53 & 0,27 & 94,40 \\
\hline 15 & J & 0,93 & 3 & 5,50 & 2,50 & 13,50 & 0,67 & 0,33 & 133,50 \\
\hline 16 & J & 0,94 & 3 & 6,73 & 3,93 & 16,27 & 0,75 & 0,37 & 140,24 \\
\hline 17 & K & 0,95 & 1 & 1,30 & 2,39 & 2,39 & 0,22 & 0,22 & 47,39 \\
\hline 18 & L & 1,00 & 2 & 0,00 & 0,00 & 0,00 & 0,00 & 0,00 & 0,00 \\
\hline 19 & L & 1,00 & 3 & 0,00 & 2,25 & 4,25 & 0,50 & 0,25 & 70,00 \\
\hline 20 & M & 0,64 & 1 & 7,00 & 8,00 & 7,00 & 1,00 & 0,50 & 100,00 \\
\hline 21 & N & 0,99 & 1 & 0,00 & 0,00 & 0,00 & 0,00 & 0,00 & 0,00 \\
\hline 22 & O & 1,00 & 1 & 0,00 & 0,00 & 0,00 & 0,00 & 0,00 & 0,00 \\
\hline 23 & P & 0,63 & 1 & 9,54 & 7,85 & 11,22 & 1,12 & 0,56 & 122,85 \\
\hline
\end{tabular}


Tabela 5 - Resumo estatístico dos aj ustes dos insumos por processo construtivo

\begin{tabular}{|c|c|c|c|c|c|c|}
\hline \multicolumn{7}{|c|}{ 1- Viga faixa protendida com lajes nervuradas } \\
\hline & X.SERV & X.AUX & X.PROF & X.ENCAR & X.MESTRE & POTSALV \\
\hline Média & 1,78 & 2,67 & 3,55 & 0,39 & 0,19 & 47,20 \\
\hline Desvio Padrão & 3,03 & 2,94 & 3,89 & 0,40 & 0,20 & 46,52 \\
\hline Máximo & 9,54 & 8,00 & 11,22 & 1,12 & 0,56 & 122,85 \\
\hline Mínimo & 0,00 & 0,00 & 0,00 & 0,00 & 0,00 & 0,00 \\
\hline \multicolumn{7}{|c|}{ 2- Laje lisa com viga de borda } \\
\hline Média & 0,00 & 3,40 & 3,40 & 0,00 & 0,20 & 46,00 \\
\hline Desvio Padrão & 0,00 & 4,81 & 4,81 & 0,00 & 0,28 & 65,05 \\
\hline Máximo & 0,00 & 6,80 & 6,80 & 0,00 & 0,40 & 92,00 \\
\hline Mínimo & 0,00 & 0,00 & 0,00 & 0,00 & 0,00 & 0,00 \\
\hline \multicolumn{7}{|c|}{3 - Laje lisa sem viga de borda } \\
\hline Média & 2,83 & 1,94 & 8,04 & 0,45 & 0,25 & 83,02 \\
\hline Desvio Padrão & 2,97 & 1,32 & 6,24 & 0,28 & 0,13 & 52,05 \\
\hline Máximo & 6,73 & 3,93 & 16,27 & 0,75 & 0,37 & 140,24 \\
\hline Mínimo & 0,00 & 0,00 & 0,00 & 0,00 & 0,00 & 0,00 \\
\hline \multicolumn{7}{|c|}{ 4- Viga alta protendida de borda e lajes nervuradas } \\
\hline Média & 0,00 & 1,75 & 1,38 & 0,25 & 0,00 & 32,50 \\
\hline Desvio Padrão & 0,00 & 2,47 & 1,94 & 0,35 & 0,00 & 45,96 \\
\hline Máximo & 0,00 & 3,50 & 2,75 & 0,50 & 0,00 & 65,00 \\
\hline Mínimo & 0,00 & 0,00 & 0,00 & 0,00 & 0,00 & 0,00 \\
\hline
\end{tabular}

\section{Conclusões e sugestões}

Este trabalho apresentou uma comparação entre sistemas estruturais para edifícios em concreto e apontou para uma escolha da estrutura com base nos critérios de eficiência e melhor uso dos insumos. Estruturas de concreto são produtos intensivos em trabalho, portanto a melhor estratégia para incrementar a eficiência desse processo é realizar uma melhor alocação da mão de obra adotada.

A amostra levantada indica potencial de redução nas equipes de profissionais da construção ou no gasto com capital em formas, desformas, etc. Entretanto, o último caso é mais sensível a mudanças, pois tem aspectos estruturais envolvidos.

Os modelos desenvolvidos indicam que há possibilidade de ganhos de escala nesses ajustes e que substituir trabalho por capital é mais vantajoso que o inverso, indicando que melhoras no processo produtivo são um caminho para ganhos de produtividade.

Como limitações do estudo, podem ser ressaltados a falta de um acompanhamento contínuo dos processos estudados, a possibilidade do uso de trabalhadores em mais de um processo e o determinismo do modelo proposto.

O trabalho empregou um modelo DEA, entretanto vários dos outros processos envolvidos na construção civil carregam peculiaridades que podem exigir o uso de métodos paramétricos ou não paramétricos mais eficientes e que repliquem mais precisamente a realidade. Portanto, sugere-se uma investigação que amplie a amostragem e avalie outros processos, procurando-se compreender o trade-off entre o processo construtivo e a produtividade/eficiência. Adicionalmente, pode-se pensar em trabalhar com funções custo, verificando ganhos monetários em ajustes na mão de obra.

\section{Referências}

ARAÚJO JUNIOR, A. F.; NOGUEIRA, D. G.; SHIKIDA, C. D. Analysis of the Efficiency of National Civil Construction Firms. Brazilian Business Review, v. 9, n. 3, p. 45 - 70, 2012.

ASSOCIAÇÃO BRASILEIRA DE NORMAS TÉCNICAS. NBR 15575: edificações habitacionais: desempenho. Rio de Janeiro, 2013.

ASSOCIAÇÃO BRASILEIRA DE NORMAS TÉCNICAS. NBR 6118: projeto de estruturas de concreto: procedimentos. Rio de Janeiro, 2014.

BANKER R. D.; CHARNES, A.; COOPER, W. W. Some Models for Estimating Technical and Scale Inefficiencies in Data Envelopment Analysis. Management Science, v. 30, n. 9, p. 1078-1092, 1984. 
BERR, L. R.; FORMOSO, C. R. Método Para Avaliação da Qualidade de Processos Construtivos em Empreendimentos Habitacionais de Interesse Social. Ambiente Construído, Porto Alegre, v. 12, n. 2, p. 77-96, abr./jun. 2012.

BRONDANI, R. P.; MOHAMAD, G.; ISAIA, G. C. Sustentabilidade em Projeto de Estrutura de Concreto Armado. Concreto \& Contrução, v. 77, p. 102-106, 2015.

CALÇADA, P. A. B. Estudo dos Processos Produtivos na Construção Civil Objetivando Ganhos de Produtividade e Qualidade. Rio de Janeiro, 2014. Monografia (Trabalho de Conclusão de Curso em Engenharia Civil) - Escola de Engenharia, Universidade Federal do Rio de Janeiro, Rio de Janeiro, 2014.

CHARNES, A.; COOPER, W. W.; RHODES, E. Measuring Efficiency of Decision Making Units. European Journal of Operational Research, v. 2, p. 429-444, 1978.

CHARNES, A. et al. Data Envelopment Analysis: theory, methodology and application. Boston: Kluwer Academic Publishers, 1994.

\section{COELLI, T. et al. A Primer on Efficiency}

Measurement For Utilities and Transport Regulators. Washington: The World Bank, 2003.

COELli, T.; RAO, D. S. P.; BATTESE, G. E. An Introduction to Efficiency and Productivity Analysis. Boston; Dordrecht; London: Kluwer, 1998.
DANTAS, J. D. F. Produtividade da Mão de

Obra: estudo de caso: métodos e tempos na indústria da construção civil no subsetor de edificações na cidade de João Pessoa-PB. João Pessoa, 2011. Monografia (Trabalho de Conclusão de Curso em Engenharia Civil) - Universidade Federal da Paraíba, João Pessoa, 2011.

FUNDAÇÃO GETÚLIO VARGAS PROJETOS.

A Produtividade da Construção Civil Brasileira. Brasília: Câmara Brasileira da Indústria da Construção, 2012.

GUJARATI, D. N.; PORTER, D. C. Econometria Básica. 5. ed. Porto Alegre: AMGH, 2011.

MCCABE, B.; TRAN, V.; RAMANI, J. Construction Prequalification Using Data Envelopment Analysis. Canadian Journal of Civil Enginering, v. 32, p. 183-193, 2005.

SILVA, F. G. F. et al. Análise Exploratória da Eficiência Produtiva dos Portos Brasileiros. Transportes, v. 19, p. 6-12, 2011.

TATARI, O.; KUCUKVAR, M. Eco-Efficiency of Construction Materials: data envelopment analysis. Journal of Construction Engineering and Management, v. 138, n. 6, p. 733-741, 2012.

WOOLDRIDGE, J. M. Introdução à

Econometria: uma abordagem moderna. 4. ed. Thomson, 2011.

\section{Gildemir Silva}

Faculdade de Economia, Administração, Atuária e Contabilidade | Universidade Federal do Ceará | Rua Marechal Deodoro, 400 | Fortaleza - CE - Brasil | CEP 60020-060 | Tel.: (85) 3231-3989 | E-mail: gildemir@gmail.com

\section{Bruno Prata}

Departamento de Engenharia de Produção | Universidade Federal do Ceará | Campus do Picí, Bloco 714 | Fortaleza - CE - Brasil | CEP 60440-554 | Tel.: (85) 3366-9633 | E-mail: baprata@ufc.br

\section{Augusto Albuquerque}

Departamento de Engenharia Estrutural e Construção Civil | Universidade Federal do Ceará | Campus do Picí, Bloco 728 | Fortaleza - CE Brasil | CEP 60455-900 | Tel.: (85) 3366-9607 | E-mail: augusto.albuquerque@ufc.br

\section{Revista Ambiente Construído}

Associação Nacional de Tecnologia do Ambiente Construído

Av. Osvaldo Aranha, 99 - 3o andar, Centro

Porto Alegre - RS - Brasil

CEP $90035-190$

Telefone: +55 (51) 3308-4084

Fax: +55 (51) 3308-4054

www. seer. ufrgs. br/ ambienteconstruido

E-mail: ambienteconstruido@ufrgs.br 